Check for updates

Cite this: RSC Adv., 2018, 8, 31755

\title{
Study of magnetic and electrical properties of $\mathrm{Pr}_{0.65} \mathrm{Ca}_{0.25} \mathrm{Ba}_{0.1} \mathrm{MnO}_{3}$ manganite
}

\author{
Ali Mleiki, (D) ${ }^{* a b}$ R. Hanen, ${ }^{a}$ H. Rahmouni, (D) ${ }^{a}$ N. Guermazi, ${ }^{c}$ K. Khirouni, ${ }^{d}$ E. K. Hlil ${ }^{e}$ \\ and A. Cheikhrouhou ${ }^{\mathrm{b}}$
}

The magnetic properties and magnetocaloric effect (MCE) in $\operatorname{Pr}_{0.65} \mathrm{Ca}_{0.25} \mathrm{Ba}_{0.1} \mathrm{MnO}_{3}$ have been investigated supplemented by electrical data. X-ray diffraction shows that the sample crystallizes in the distorted orthorhombic system with the Pnma space group. $\operatorname{Pr}_{0.65} \mathrm{Ca}_{0.25} \mathrm{Ba}_{0.1} \mathrm{MnO}_{3}$ undergoes paramagneticferromagnetic (PM-FM) phase transition at $T_{C} \sim 85 \mathrm{~K}$. For a magnetic field change of $5 \mathrm{~T}$, the maximum value of the magnetic entropy change $\left(-\Delta S_{M}^{\max }\right)$ is estimated to be $4.4 \mathrm{~J} \mathrm{~kg}^{-1} \mathrm{~K}^{-1}$ around $T_{C}$ with a large relative cooling power (RCP) value of $263.5 \mathrm{~J} \mathrm{~kg}^{-1}$. While the modified Arrott plots suggested that the magnetic transition belongs to the second order phase transitions, the universal curves of the rescaled magnetic entropy $\left(\Delta S_{M}\right)$ proved the opposite. The electrical properties of $\operatorname{Pr}_{0.65} \mathrm{Ca}_{0.25} \mathrm{Ba}_{0.1} \mathrm{MnO}_{3}$ have been investigated using impedance spectroscopy techniques. The dc-resistivity $\left(\sigma_{\mathrm{dc}}\right)$ study shows the presence of semiconductor behavior. Ac-conductivity $\left(\sigma_{\mathrm{ac}}\right)$ analysis shows that the conductivity is governed by a hopping process. From the analysis of the alternating regime, the exponent $s$ variation obtained is in good agreement with Mott theory. The impedance spectrum analysis reveals the presence of a relaxation phenomenon. Based on these analyzes, the sample can be modeled by an electrical equivalent circuit.

Received 5th July 2018

Accepted 4th September 2018

DOI: $10.1039 / \mathrm{c} 8 \mathrm{ra} 05747 \mathrm{~h}$

rsc.li/rsc-advances $\left\langle r_{\mathrm{A}}\right\rangle$ in the A-site and the A-site cationic disorder. Introducing quenched disorder in manganites as different ions at A-site can leads to a change in structure, which modifies the original system completely. A-site doping can be strongly influenced by the average ionic radius, which exhibits a close relation between the distortion of $\mathrm{MnO}_{6}$ octahedra and the narrowing of the electronic bandwidth.

The low bandwidth manganites $\operatorname{Pr}_{1-x} \mathrm{Ca}_{x} \mathrm{MnO}_{3}$ have been the subject of several studies with calcium concentration due to their diverse physical properties such as charge-ordering $(\mathrm{CO}),{ }^{5}$ metamagnetic transition, ${ }^{6}$ colossal magnetoresistance ${ }^{7,8}$ and large magnetocaloric effects. ${ }^{9}$ In the case of a $35 \%$ Ca-doped sample, ${ }^{10}$ it undergoes a charge-ordering (CO) transition at about $230 \mathrm{~K}$, an antiferromagnetic (AFM) transition in the vicinity of $160 \mathrm{~K}$. Without applied magnetic field, its electrical conduction shows insulator characteristics. Below the AFM transition temperature, application of a high magnetic field (above $\mu_{0} H=4 \mathrm{~T}$ ) leads this one to metallic and ferromagnetic (FM) state. In addition, it is worth mentioning for the $\operatorname{Pr}_{0.65}(-$ $\left.\mathrm{Ca}_{1-x} \mathrm{Sr}_{x}\right)_{0.35} \mathrm{MnO}_{3}$ compounds the partial substitution of $\mathrm{Sr}^{2+}$ ions for $\mathrm{Ca}^{2+}$ ions, having higher ionic radius, shows up many physical interesting phenomena, ${ }^{11-13}$ particularly with $x \approx 0.3$ the charge and orbital ordering appear below $200 \mathrm{~K}$, but above $x$ $\approx 0.3$ collapse into the metallic state has been observed below $100 \mathrm{~K}^{14}$

Study of A-site doped $\mathrm{Pr}_{0.65} \mathrm{Ca}_{0.35} \mathrm{MnO}_{3}$ sample would offer a complementary understanding of the structural, magnetic France 
and electrical properties. To the best of our knowledge, there are no reports on the physical properties of $\mathrm{Ba}$ doped $\mathrm{Pr}_{0.65^{-}}$ $\mathrm{Ca}_{0.35} \mathrm{MnO}_{3}$ compounds. Substitution of calcium by barium is interesting as the difference between ionic sizes of $\mathrm{Ca}$ and $\mathrm{Ba}$ is significantly large. The selection of $\mathrm{Ba}$ as a dopant was inspired by an intention to induce chemical pressure inside the lattice to favor the electron/hole transfer between the neighboring $\mathrm{Mn}$ sites. In this context, we report how a direct substitution of barium ion in the A-site affects the physical properties as compared to pure $\operatorname{Pr}_{0.65} \mathrm{Ca}_{0.35} \mathrm{MnO}_{3}$. Our results show that at $x$ $=0.10$ doping level the charge ordering $(\mathrm{CO})$ is destroyed and drastic changes of properties have been observed.

\section{Experimental details}

Polycrystalline perovskite $\operatorname{Pr}_{0.65} \mathrm{Ca}_{0.25} \mathrm{Ba}_{0.1} \mathrm{MnO}_{3}$ sample was fabricated by standard solid-state reaction method. After thoroughly mixing the stoichiometric reactants of high purity (99.99\%) [Aldrich] $\mathrm{Pr}_{6} \mathrm{O}_{11}, \mathrm{CaCO}_{3}, \mathrm{BaCO}_{3}$ and $\mathrm{MnO}_{2}$, the sample is fired at $900{ }^{\circ} \mathrm{C}$ for $12 \mathrm{~h}$. Then, the mixture was pressed into pellets and sintered at 1000-1200 K for $48 \mathrm{~h}$ in air with several intermediate grindings. The powder $\mathrm{X}$-ray diffractometer ("Panalytical X pert Pro" diffractometer with $\mathrm{Cu}-\mathrm{K} \alpha$ radiation $(\lambda$ $=1.5406 \AA$ )) was employed to identify the phase purity and crystalline structure at room temperature. The unit cell parameters were refined using the Rietveld analysis of the X-ray powder diffraction data with the help of FULLPROF software. ${ }^{15}$ The static magnetic measurements were carried out in magnetic fields up to $5 \mathrm{~T}$ at temperatures between 5 and $285 \mathrm{~K}$ on a BS2 magnetometer equipped with a super conducting coil and developed at Neel Institute. On the both side of the pellet, we deposited a thin aluminum film through a circular mask of $6 \mathrm{~mm}$ of diameter. Then, we obtained a configuration of a plate capacitor used to measure both the capacitance and the conductance. The sample is mounted in a cryostat which allows the variation of temperature from 80 to $300 \mathrm{~K}$. Conductance and capacitance measurement are performed using an Agilent 4294A analyzer.

\section{Results and discussion}

\section{(a) X-ray diffraction}

In order to determine the crystalline structure of $\mathrm{Pr}_{0.65} \mathrm{Ca}_{0.25^{-}}$ $\mathrm{Ba}_{0.1} \mathrm{MnO}_{3}$, room temperature powder X-ray diffraction (XRD) pattern was collected. The XRD pattern of $\operatorname{Pr}_{0.65} \mathrm{Ca}_{0.25} \mathrm{Ba}_{0.1} \mathrm{MnO}_{3}$ sample is shown in Fig. 1. It is seen from the results that all peaks can be indexed according to a perovskite structure with orthorhombic Pnma symmetry. Rietveld refinement confirms that there are no detectable impurities in this compound. From the XRD pattern lattice parameters obtained from refinements are: 5.4565(3) $\mathrm{A}, b=7.7240(4) \AA$ and $c=5.4519(5) \AA$.

\section{(b) Magnetic and magnetocaloric effect}

The temperature dependence of the magnetization, $M(T)$, measured via field-cooled (FC) protocol at $0.05 \mathrm{~T}$ applied magnetic field is shown in Fig. 2(a). With decreasing

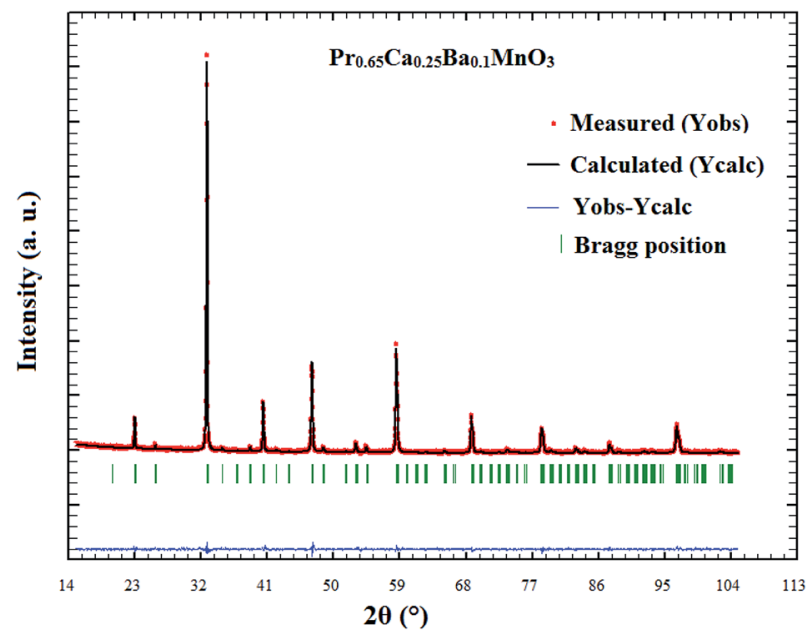

Fig. 1 Rietveld refined powder XRD patterns of the $\operatorname{Pr}_{0.65} \mathrm{Ca}_{0.25} \mathrm{Ba}_{0.1^{-}}$ $\mathrm{MnO}_{3}$ compound.
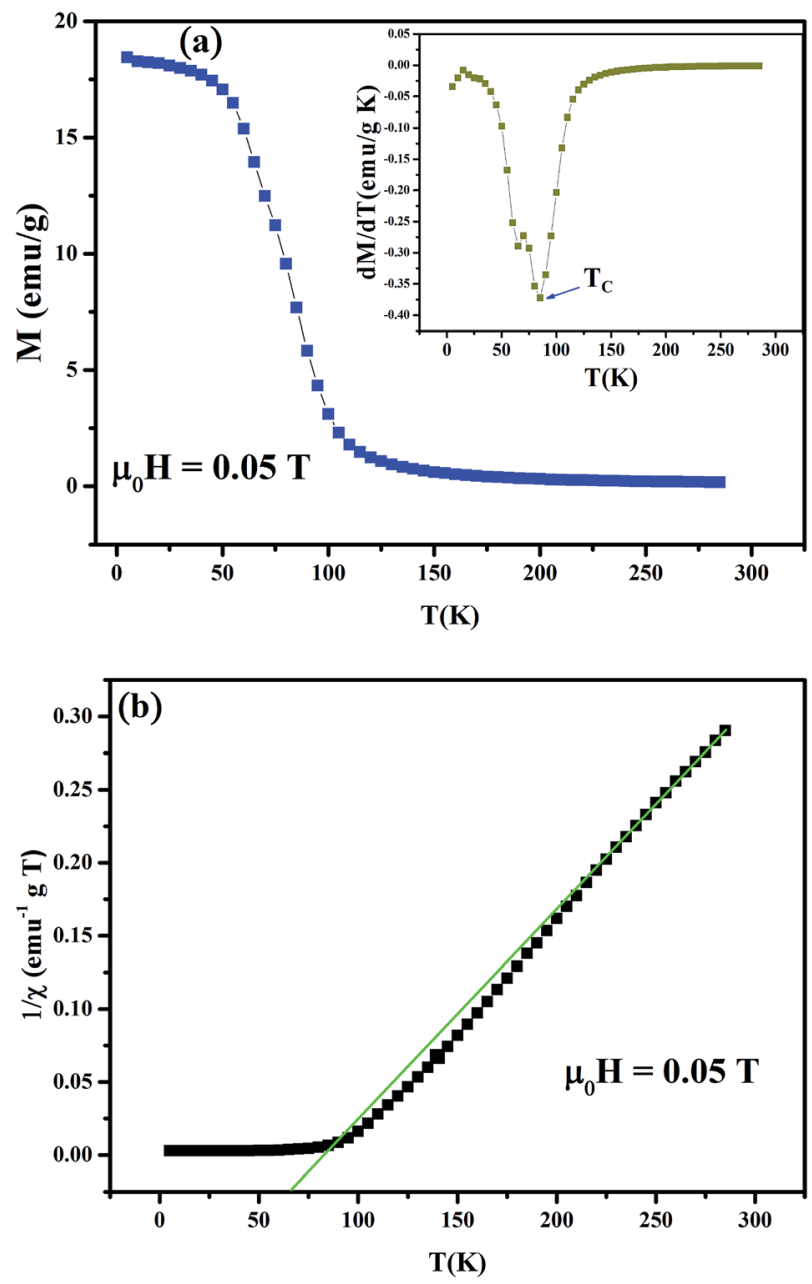

Fig. 2 (a) Temperature dependence of FC magnetization measured at a field of $0.05 \mathrm{~T}$, the inset shows the derivative of magnetization with respect to temperature ( $\mathrm{d} M / \mathrm{d} T$ vs. $T$ ). (b) Inverse susceptibility versus temperature plot for $\operatorname{Pr}_{0.65} \mathrm{Ca}_{0.25} \mathrm{Ba}_{0.1} \mathrm{MnO}_{3}$ bulk. 
temperature the $\mathrm{Pr}_{0.65} \mathrm{Ca}_{0.25} \mathrm{Ba}_{0.1} \mathrm{MnO}_{3}$ compound displays a typical paramagnetic-ferromagnetic (PM-FM) transition. The Curie temperature $\left(T_{\mathrm{C}}\right)$, corresponding to the minimum in $\mathrm{d} M$ / $\mathrm{d} T-T$ curve (see inset of Fig. 2(a)), is determined to be $85 \mathrm{~K}$. Apparently, as compared to $\mathrm{Pr}_{0.65} \mathrm{Ca}_{0.35} \mathrm{MnO}_{3},{ }^{16}$ a $10 \%$ barium substitution at the A-site destroys the charge ordering (CO) in the pristine compound. Because the larger ionic size of $\mathrm{Ba}^{2+}$ compared to that of $\mathrm{Ca}^{2+}$, the destruction of $\mathrm{CO}$ state can be attributed to the increase of the average ionic radius of the Asite $\left(\left\langle r_{\mathrm{A}}\right\rangle\right)$ which leads to the increase of $\mathrm{e}_{\mathrm{g}}$ bandwidth, favors the electrons movement through the lattice and promotes the FM metallic state. The isoelectronic A-site substitution controls the structure by the distortion of $\mathrm{MnO}_{6}$ octahedra, i.e., tilting and rotation of the $\mathrm{MnO}_{6}$ octahedra, which modifies local structural parameters such as the $\mathrm{Mn}-\mathrm{O}$ bond distance and Mn-O-Mn bond angle, and then directly influence the case of electron hopping between Mn ions. ${ }^{17}$ Several works on manganites show that the FM state as well as the CO are affected due to the chemical pressure at A-site. ${ }^{18-20}$

In Fig. 2(b) we have reported the inverse susceptibility $1 / \chi$ as a function of temperature for $\operatorname{Pr}_{0.65} \mathrm{Ca}_{0.25} \mathrm{Ba}_{0.1} \mathrm{MnO}_{3}$. A deviation has been observed which starts around $225 \mathrm{~K}$ far above $T_{\mathrm{C}}$, which may be due to growth of short range magnetic interactions in the compound. ${ }^{17,19}$ However, the curve in PM regime from 230 to $285 \mathrm{~K}$ obeys the Curie-Weiss (CW) formula $\chi=C /\left(T-\theta_{\mathrm{P}}\right)$, where $C$ is the Curie constant and $\theta_{\mathrm{P}}$ is the PM Curie temperature. The fitting of the linear region to the CW law yields positive $\theta_{\mathrm{P}}, \theta_{\mathrm{P}}=66.5 \mathrm{~K}$, indicating dominant $\mathrm{FM}$ interactions. On another hand, the detailed calculation of the theoretical effective moment $\left(\mu_{\text {eff }}^{\text {theo }}\right)$ can be found elsewhere. ${ }^{21}$ Moreover, the experimental effective moment is found to be $\mu_{\text {eff }}^{\exp }=14.5 \mu_{\mathrm{B}}$, which is much larger than its theoretical counterpart $\mu_{\text {eff }}^{\text {theo }}=5.53 \mu_{\mathrm{B}}$, confirming clearly the magnetic inhomogeneity of our sample. This behavior can be explained in terms of the presence of FM clusters of $\mathrm{Mn}^{3+}, \mathrm{Mn}^{4+}$ double exchange pairs together in the PM region ${ }^{22}$

Fig. 3(a) shows the evolution of isothermal magnetization as a function of the magnetic applied field, $M(H)$, for $\mathrm{Pr}_{0.65} \mathrm{Ca}_{0.25^{-}}$ $\mathrm{Ba}_{0.1} \mathrm{MnO}_{3}$ sample. At low temperature, we can see that magnetization increases sharply with the applied field for $\mu_{0} H<$ $0.5 \mathrm{~T}$ indicating the presence of FM behavior. The magnetic nature of the sample above $156 \mathrm{~K}$ is clearly PM as the magnetization increases linearly with increasing magnetic field.

Based on the Arrott plots, the isotherms of $M^{2}$ as a function of $H / M$ are shown in Fig. 3(b). The order of the magnetic transition was analyzed based in the criterion suggested by Banerjee. ${ }^{23}$ As can be observed, the positive slopes of the Arrott plots for $\mathrm{Pr}_{0.65} \mathrm{Ca}_{0.25} \mathrm{Ba}_{0.1} \mathrm{MnO}_{3}$ sample indicate a second-order magnetic transition. According to the mean field theory, the Arrott plots should give a series of parallel straight lines around $T_{\mathrm{C}}{ }^{24}$ However, all $M^{2}$ versus $H / M$ curves show non-linear behavior in high-magnetic field having downward curvature which indicate the non-mean-field magnetic interactions in this sample.

The magnetocaloric effect (MCE) can be related to the magnetic properties of $\operatorname{Pr}_{0.65} \mathrm{Ca}_{0.25} \mathrm{Ba}_{0.1} \mathrm{MnO}_{3}$. The MCE have been estimated from the temperature and field dependence of
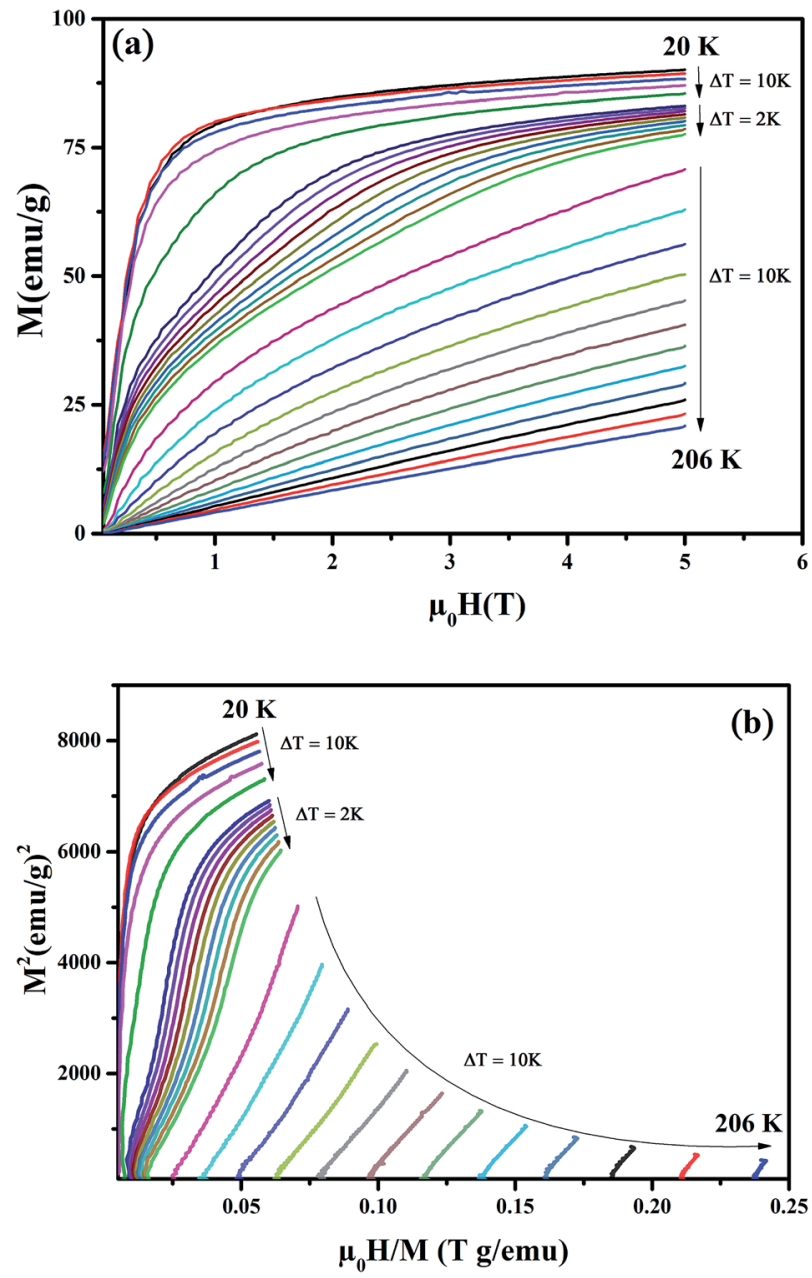

Fig. 3 (a) Isothermal magnetization around $T_{C}$ for $\operatorname{Pr}_{0.65} \mathrm{Ca}_{0.25} \mathrm{Ba}_{0.1^{-}}$ $\mathrm{MnO}_{3}$ compound at different temperatures. (b) Standard Arrott plots (isotherms $M^{2}$ vs. H/M).

the magnetization $M(H, T)$, (see Fig. 3(a)), through the thermodynamics Maxwell's relation: ${ }^{25}$

$$
\Delta S_{\mathrm{M}}(T, H)=S_{\mathrm{M}}\left(T, H_{1}\right)-S_{\mathrm{M}}\left(T, H_{2}\right)=\int_{H_{1}}^{H_{2}}\left(\frac{\partial M}{\partial T}\right) \mathrm{d} H
$$

It can be found that the maximum value of magnetic entropy $\left(-\Delta S_{\mathrm{M}}\right)$ increases monotonically with increasing magnetic field change. In addition, we can see in Fig. 4(a) the presence of a transition temperature shift induced by the applied magnetic field. Therefore, the $-\Delta S_{\mathrm{M}}$ is maximized around $T_{\mathrm{C}}$ and its maximum values $\left(-\Delta S_{\mathrm{M}}^{\max }\right)$ are found to be approximately 2.5 and $4.4 \mathrm{~J} \mathrm{~kg}^{-1} \mathrm{~K}^{-1}$ under a magnetic field changes of 2 and $5 \mathrm{~T}$. Other important parameter of refrigerant materials is the relative cooling power (RCP) which is defined by the product of the peak entropy change and the full width at half maximum of the peak, i.e., RCP $=-\Delta S_{\mathrm{M}}^{\max } \delta_{\text {TFWHM }}$ (where $\delta_{\text {TFWHM }}$ is the full width at half-maximum of $\left.\Delta S_{\mathrm{M}}(T)\right)$. Thus, the resulting RCP values are found to be 104.2 and $263.5 \mathrm{~J} \mathrm{~kg}^{-1}$ under a magnetic field changes of 2 and $5 \mathrm{~T}$, respectively. The obtained values are 

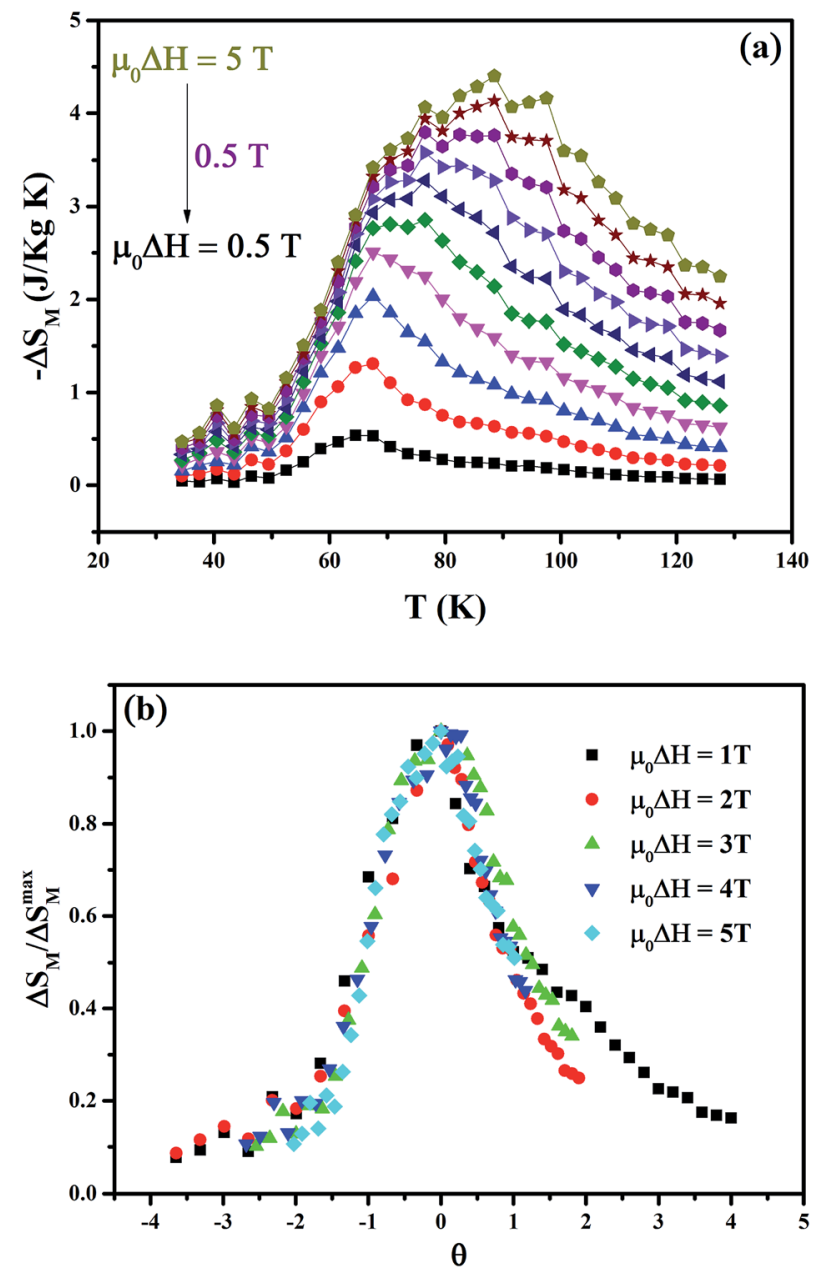

Fig. 4 (a) Temperature dependence of the magnetic entropy changes $\Delta S_{M}$ under various external magnetic fields. (b) Universal curves of $\operatorname{Pr}_{0.65} \mathrm{Ca}_{0.25} \mathrm{Ba}_{0.1} \mathrm{MnO}_{3}$ sample.

comparable with those of several manganite systems with a large MCE, ${ }^{26-32}$ suggesting that our compound may be suitable candidate for magnetic refrigeration. The large MCE in $\mathrm{Pr}_{0.65^{-}}$ $\mathrm{Ca}_{0.25} \mathrm{Ba}_{0.1} \mathrm{MnO}_{3}$ can be understood by its sharp magnetization change at the FM-PM transition and its high magnetization saturation.

Additionally, a phenomenological universal curve for $\Delta S_{\mathrm{M}}(T)$ in the vicinity of a second-order phase transition has been theoretically established ${ }^{33,34}$ and successfully applied to several families of magnetic material, ${ }^{35-38}$ which can be used as a further criterion to reveal the order of the magnetic phase transition. In addition, this scaling can be particularly helpful for investigating the refrigerant capacity of a similar material. However, a breakdown of the universal curve can be attributed to inhomogeneity within the material. ${ }^{39}$ The temperature axis was transformed according to the relation:

$$
\theta=\left\{\begin{array}{l}
-\left(T-T_{\mathrm{c}}\right) /\left(T_{1}-T_{\mathrm{c}}\right) \quad T \leq T_{\mathrm{c}} \\
\left(T-T_{\mathrm{c}}\right) /\left(T_{2}-T_{\mathrm{c}}\right) \quad T>T_{\mathrm{c}}
\end{array}\right.
$$

where $T_{1}$ and $T_{2}$ are the temperature of the two reference points. For present study, $T_{1}$ and $T_{2}$ are chosen such that $\Delta S_{\mathrm{M}}\left(T_{1}\right) /$
$\Delta S_{\mathrm{M}}^{\max }=\Delta S_{\mathrm{M}}\left(T_{2}\right) / \Delta S_{\mathrm{M}}^{\max }=0.5$. The rescaled $\Delta S_{\mathrm{M}}(T)$ curves for different maximum applied magnetic fields ranging from 1 up to $5 \mathrm{~T}$ with increments of $1 \mathrm{~T}$ are shown in Fig. 4(b). It has been reported that the essential region for collapse to determine the order of the transition is $\theta<-1\left(T<T_{\mathrm{C}}\right)$. However, the deviation of collapse for first-order phase transition may not be obvious around $-1<\theta<0$. The divergence of the curves is clear in the $\mathrm{Pr}_{0.65} \mathrm{Ca}_{0.25} \mathrm{Ba}_{0.1} \mathrm{MnO}_{3}$ compound, particularly far above and below the $T_{\mathrm{C}}$, indicating clearly that this compound does not follows an universal curve and the magnetic phase transition is first order. Thus, we conclude that there is not perfect agreement of the rescaled curves below $T_{\mathrm{C}}$, however a check of the Arrott plots $\left(M^{2}(H / M)\right)$ constructions suggested the second order quality of the transition in our sample.

\section{(c) DC-resistivity study}

The variation of the dc-resistivity $(\rho)$ as a function of temperature of $\mathrm{Pr}_{0.65} \mathrm{Ca}_{0.25} \mathrm{Ba}_{0.1} \mathrm{MnO}_{3}$ is represented schematically in Fig. 5. Across all the studied temperature ranges, the $\rho$ decreases by increasing temperature which indicates that this compound exhibits a semiconductor behavior. The decrease in $\rho$ when the temperature increases could be due to the increase the density of free carriers that acquire a sufficient thermal energy thermal energy to easily cross the barriers encountered.

\section{(d) AC-conductivity study}

The inset of Fig. 5 shows the frequency dependence of conductivity $(\sigma)$ at different temperatures for $\operatorname{Pr}_{0.65} \mathrm{Ca}_{0.25} \mathrm{Ba}_{0.1^{-}}$ $\mathrm{MnO}_{3}$. As we can observe, ac-conductivity $\left(\sigma_{\mathrm{ac}}\right)$ spectrum exhibits the presence of several behaviors. At low frequency $(f<$ $\left.10^{4} \mathrm{~Hz}\right)$, a large plateau, corresponds to dc-conductivity $\left(\sigma_{\mathrm{dc}}\right)$, appears and the $\sigma$ increases by increasing temperature. Such behavior indicates that conduction process is thermally activated by increasing the density of free carriers in the structure. Above $10^{5} \mathrm{~Hz}$ and for $T>180 \mathrm{~K}$, a metallic behavior appears

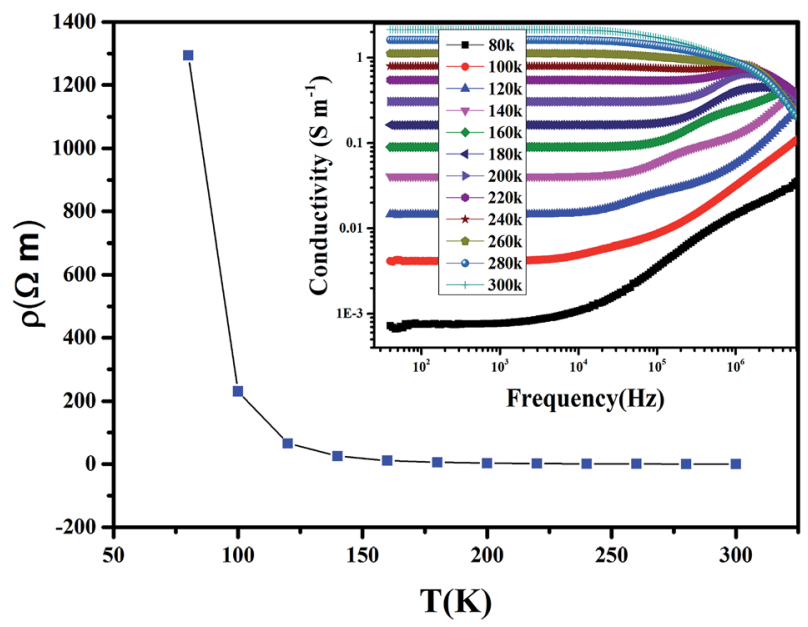

Fig. 5 Variation of the electrical resistivity $(\rho)$ versus temperature deduced from the dc conductivity $\sigma_{\mathrm{dc}}(T)$, the inset shows the variation of conductivity $(\sigma)$ as a function of frequency at various temperatures for $\operatorname{Pr}_{0.65} \mathrm{Ca}_{0.25} \mathrm{Ba}_{0.1} \mathrm{MnO}_{3}$. 
accompanied by a decrease in $\sigma$. However, for $T<180 \mathrm{~K}$ the $\sigma$ monotonically increases with increasing frequency, indicating a superposition of different relaxation processes. ${ }^{40}$ The range of temperature, where $\sigma$ monotonically increases, could be described by the law of Jonscher:

$$
A=\omega^{s}
$$

where $A$ is a constant determining the strength of polarizability and $s$ is the frequency exponent representing the degree of interaction between mobile charge and the lattices around them. To get an information about the conduction mechanism in our sample, we will study the variation of the exponent $s$ depending on the temperature. From the region where the $\sigma$ is proportional to $\omega^{s}$ the value of the exponent $s$ is determined from the slope of the linear parts of $\sigma$ versus frequency. As shown in Fig. 6(a), the exponent $s$ decreases by increasing temperature. The result are in good agreement with a hopping mechanism in disordered material ${ }^{\mathbf{4 1}}$ and confirm that conduction process is thermally activated. Such behavior is observed in different oxides and amorphous compounds and is discussed using Pike model. ${ }^{42}$ Also such variation is in good agreement with the model of Mott and Davis: ${ }^{43}$
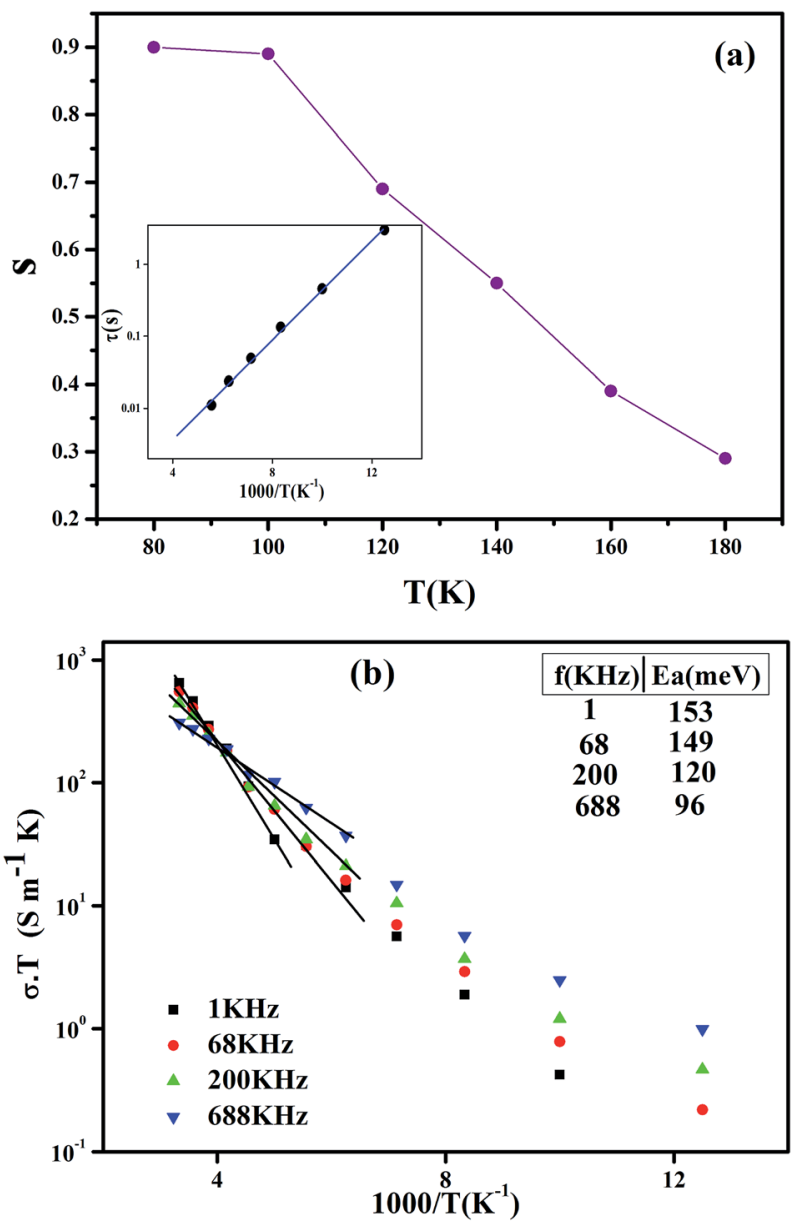

Fig. 6 (a) Temperature dependence of the frequency exponent $s$, the inset shows the plot of ' $\tau$ ' versus 1000/T. (b) Arrhenius plot of $\left(\sigma_{\mathrm{ac}} T\right)$ at different frequencies for $\operatorname{Pr}_{0.65} \mathrm{Ca}_{0.25} \mathrm{Ba}_{0.1} \mathrm{MnO}_{3}$.

$$
s=1-\left(6 k_{\mathrm{B}} T / W_{\mathrm{m}}\right)
$$

where $k_{\mathrm{B}}$ is a Boltzmann's constant, $T$ is the absolute temperature and $W_{\mathrm{m}}$ is defined as the energy required for an electron to pass from one site to another. The observed decrease in the frequency exponent $s$ with increasing temperature suggests that the correlated barrier hopping $(\mathrm{CBH})$ model best describes the ac conduction mechanism. ${ }^{44}$ In the $\operatorname{Pr}_{0.65} \mathrm{Ca}_{0.25} \mathrm{Ba}_{0.1} \mathrm{MnO}_{3}$ compound, large number of crystal boundaries acts as barriers which are overcome by the charge carriers, resulting in conduction across the samples. According to Funke ${ }^{45}$ in Joncher's equation, if $s \leq 1$, it indicates that the hopping motion due to translational motion.

Fig. 6(b) shows the product 'conductivity temperature' $(\sigma T)$ versus $(1000 / T)$ at different frequencies $(1 \mathrm{kHz}, 68 \mathrm{kHz}, 200 \mathrm{kHz}$ and $688 \mathrm{kHz}$ ). The high temperature conductance data were well fitted to the following equation: ${ }^{43}$

$$
\sigma=\sigma_{0} / T \exp \left(-E_{\mathrm{ac}} / k_{\mathrm{B}} T\right)
$$

where $\sigma_{0}$ is a pre-exponential factor, $T$ is the absolute temperature, $E_{\text {ac }}$ represents the activation energy of conduction mechanism and $k_{\mathrm{B}}$ is the Boltzmann constant. It is clear, at high temperatures, that $\sigma$ is follows this equation, suggesting that the electronic conduction is dominated by thermally activated hopping of small polaron (SPH). ${ }^{46}$ From this figure, it is found that activation energy decreases when increasing frequency (see the inset of Fig. 6(b)). This can be explained by the fact that the charge carriers choose the easiest path between sites. ${ }^{47}$ Therefore, the hops become only between closer sites and the activation energy decreases with frequency.

\section{(e) Complex impedance analysis}

The complex impedance $Z^{*}$ of the network is defined as:

$$
Z^{*}=Z^{\prime}(\omega)+j Z^{\prime \prime}(\omega)
$$

where $Z^{\prime}$ is the real part and $Z^{\prime \prime}$ the imaginary part of the complex impedance. Fig. 7(a) displays the evolution of $Z^{\prime}$ with frequency at different temperatures for $\operatorname{Pr}_{0.65} \mathrm{Ca}_{0.25} \mathrm{Ba}_{0.1} \mathrm{MnO}_{3}$. At low frequencies, the spectrum of $Z^{\prime}$ has higher values. Such behavior is widely observed in perovskite systems. ${ }^{\mathbf{4 8 , 4 9}}$ At higher frequencies, however, the value of $Z^{\prime}$ merges for all temperatures. Such observation indicates the presence of space charge polarization, ${ }^{50}$ which is released at high temperatures. At low frequencies and when temperature increases, $Z$ ' decreases. This behavior can be related mainly to the lowering in the density of trapped charges and then a rise in the mobility of charges carriers. ${ }^{51}$ The behavior of $Z^{\prime}$ observed for this compound at lower and higher frequencies is in good agreement with the reported results in the literature. ${ }^{52,53}$ The variation of $Z^{\prime \prime}$ as a function of frequency and temperature is shown in Fig. 7(b). This spectrum is characterized by the presence of a peak at a specific frequency, which is conventionally known as the "relaxation frequency" $\left(f_{\mathrm{r}}\right)$. When the temperature increases, the relaxation frequency shifts to the higher frequency region (see inset of Fig. 7(b)). This evolution indicated the presence of 

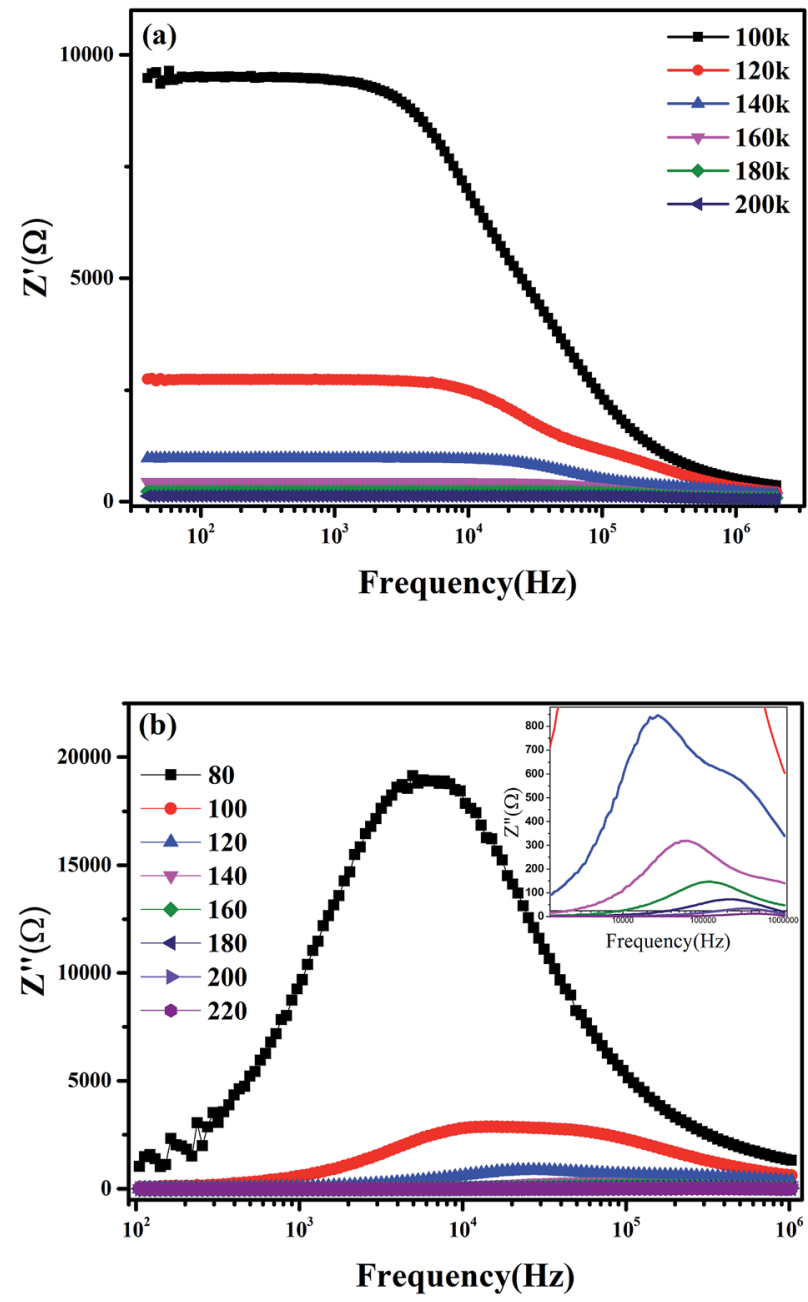

Fig. 7 (a) Frequency dependence of the real part $\left(Z^{\prime}\right)$ of the impedance at different temperatures. (b) Frequency dependence of the imaginary part $\left(Z^{\prime \prime}\right)$ of the impedance at different temperatures for $\operatorname{Pr}_{0.65} \mathrm{Ca}_{0.25^{-}}$ $\mathrm{Ba}_{0.1} \mathrm{MnO}_{3}$, The inset shows zoom-in of the high-temperature region.

a relaxation phenomenon in the studied compound. ${ }^{54}$ In addition, it is noticed that the center of each peak, i.e., at the maximum of $Z^{\prime \prime}$, does not lie at the identical frequency indicating a deviation from the typical Debye's model in the material. ${ }^{55}$ Also, it indicates that the peak intensity of $Z^{\prime \prime}$ decreases with temperature, confirming the semiconductor in this compound. ${ }^{48}$ By determining the value of the relaxation frequency, which determined for the low frequency peak centered at $f_{\mathrm{r}}$, and using the following relation: $2 \pi f_{\mathrm{r}} \tau=1$, the relaxation time $(\tau)$ can be calculated. Using Arrhenius model, the inset of Fig. 6(a) exhibits the variation of $\log \tau$ versus 1000/T. It is observed that the value of $\tau$ decreases by increasing the temperature, which indicate a thermally activated process. This result shows the presence of one conduction mechanism where the slope does not changes across all the studied temperature ranges.

Fig. 8 shows the Nyquist diagram of the system $\operatorname{Pr}_{0.65^{-}}$ $\mathrm{Ca}_{0.25} \mathrm{Ba}_{0.1} \mathrm{MnO}_{3}$ obtained at $T=80 \mathrm{~K}$. The spectrum is characterized by the appearance of a semicircle arc. To connect the

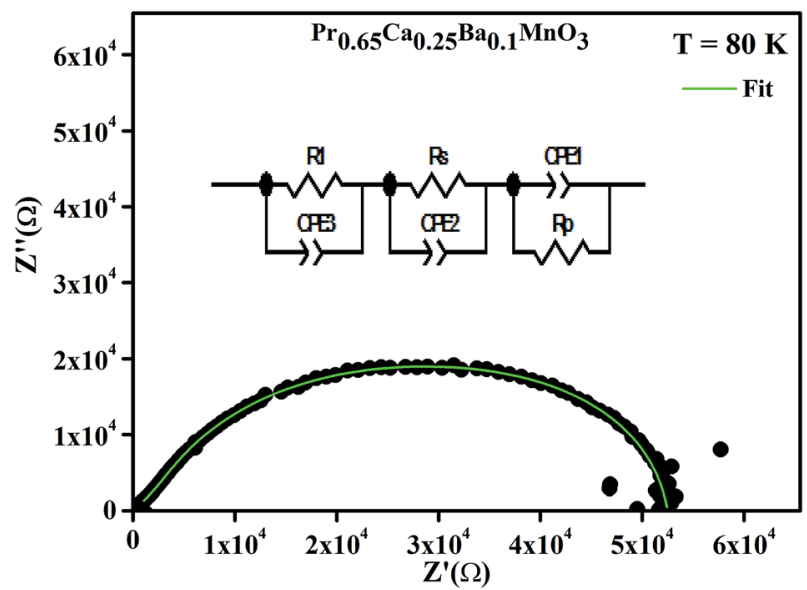

Fig. 8 Complex impedance spectrum (Nyquist plot) at $T=80 \mathrm{~K}$ with the electrical equivalent circuit.

microstructure of the compound with its electrical response, an equivalent electrical circuit can be established by modelling the impedance data using Zview software. As can be seen in Fig. 8, the agreement between the experimental and theoretical data is satisfactory. Then, the equivalent configuration is of the type $\left(\left(R_{1} / / \mathrm{ZCPE}_{3}\right)+\left(R_{\mathrm{S}} / / \mathrm{ZCPE}_{2}\right)+\left(R_{\mathrm{p}} / / \mathrm{ZCPE}_{1}\right)\right)$ as shown in the inset of Fig. 8.

\section{(f) Dielectric properties}

The study of the dielectric properties is another important source of information regarding the conduction process. The complex dielectric permittivity according to the Mott's theory ${ }^{\mathbf{4 3}}$ is defined as:

$$
\varepsilon^{*}=\varepsilon^{\prime}(\omega)-j \varepsilon^{\prime \prime}(\omega)
$$

where $\varepsilon^{\prime}$ and $\varepsilon^{\prime \prime}$ are the real and imaginary parts of $\varepsilon^{*}$, respectively. $\varepsilon^{\prime}$ describes the stored energy and $\varepsilon^{\prime \prime}$ describes the dissipated energy in the dielectric material, due to the presence of an applied electric field. The dissipation factor $(D)$ and the capacitance $(C)$ of the compound are measured over a wide frequency range $\left(40 \mathrm{~Hz}\right.$ to $10 \mathrm{MHz}$ ). Thus, $\varepsilon^{\prime}$ and $\varepsilon^{\prime \prime}$ can be determined using the following expressions: $\varepsilon^{\prime}=C t /\left(\varepsilon_{0} A\right)$ and $\varepsilon^{\prime \prime}$ $=\varepsilon^{\prime} D$, where $\varepsilon_{0}$ is the free space permittivity, $t$ is the thickness of the sample and $A$ is the area of the electrodes. The evolution of $\varepsilon^{\prime}$ and $\varepsilon^{\prime \prime}$ with frequency in the temperature range 80-160 $\mathrm{K}$ for $\mathrm{Pr}_{0.65} \mathrm{Ca}_{0.25} \mathrm{Ba}_{0.1} \mathrm{MnO}_{3}$ are shown in Fig. 9(a and b). As can be seen, at low frequencies, $\varepsilon^{\prime}$ has higher values which can be related to the presence of space charge produced via the electrode-sample contact and interface between the grain and their boundaries. ${ }^{56}$ According to Singh et al. ${ }^{57}$ and Kar et al., ${ }^{58}$ such behavior can be attributed to the presence of several parameters of polarizations such as dipolar, atomic, ionic, interfacial and electronic polarization in the compound. Therefore, under the applied alternative electric field all electric dipole moments will be oriented in his direction. Then, all the polarization mechanisms can participate to polarization of the system. At higher frequencies, the decrease in the permittivity $\varepsilon^{\prime}$ 

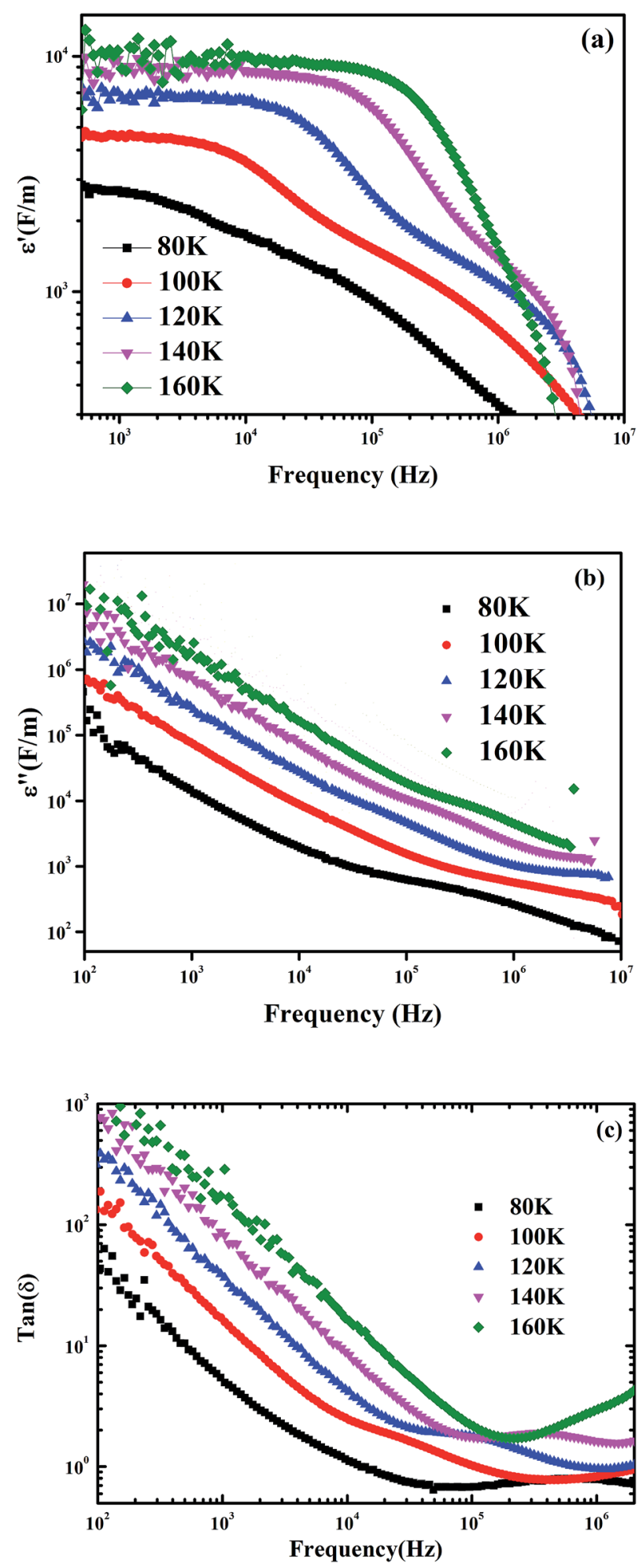

Fig. 9 Variation of real part (a) and imaginary part (b) of permittivity as a function of frequency, (c) variation of loss tangent with frequency at different temperature for $\operatorname{Pr}_{0.65} \mathrm{Ca}_{0.25} \mathrm{Ba}_{0.1} \mathrm{MnO}_{3}$.

could be explained that the heavy particles cannot follow the applied electric field and then dipole response is weak, inducing a reduction of ionic and dipolar polarization contributions. Such behavior is observed when the charge carrier hopping cannot follow the alternating current beyond a certain frequency, which decreases the probability of electrons reaching the grain boundary and as a result, polarization decreases. However, at high frequencies, $\varepsilon^{\prime}$ remains important which may be due to a localized accumulation of charges involving the presence of interfacial polarization..$^{59}$

Fig. 9(b) clearly shown that $\varepsilon^{\prime \prime}$ decreases with increasing the frequency and no peak is observed, confirming that the polarization phenomenon in this sample is governed by a hopping process. ${ }^{60}$ This result may be due to space charge polarization and interface effects. ${ }^{61}$ At low frequencies, $\varepsilon^{\prime \prime}$ has higher values, suggesting the contribution of all polarization effects particularly the interfacial/space polarization due to an inhomogeneous dielectric structure. At high frequencies, where the electric dipoles cannot follow the applied electric field, the low value attained may be due to the gradual decrease of the dielectric loss. On another hand, the variation of $\operatorname{tang}(\delta)\left(=\varepsilon^{\prime \prime} / \varepsilon^{\prime}\right)$ as a function of frequency is shown in Fig. 9(c). At constant frequency the dielectric loss increases by increasing temperature which may be due to the increase in conductivity. However, by increasing frequency, the loss tangent first decreases and then increases. Since the charge carriers cannot follow the electric field, at higher frequencies, the value of $\tan g(\delta)$ remains low.

\section{Conclusion}

Structural, magnetic and electrical properties of $\mathrm{Pr}_{0.65} \mathrm{Ca}_{0.25^{-}}$ $\mathrm{Ba}_{0.1} \mathrm{MnO}_{3}$ manganite were investigated. A Rietveld analysis shows that the sample crystallizes in the orthorhombic structure with Pnma space group. Magnetic measurements show a sharp paramagnetic to ferromagnetic transition around Curie temperature $T_{\mathrm{C}}$. A large maximum of magnetic entropy change $\left(-\Delta S_{\mathrm{M}}^{\max }\right)$ value has been found with a large relative cooling power (RCP) value. The variation of resistivity as a function of the temperature shows the presence of a semiconductor behavior. The ac-conductivity study is obeyed to the Jonscher law. The dominance of hopping mechanism was confirmed by the variation of the exponent $s$. Complex impedance analysis permits to model the sample by an electrical equivalent circuit and displays the presence of a relaxation phenomenon. The dielectric study shows the contribution of different types of polarization.

\section{Conflicts of interest}

There are no conflicts to declare.

\section{References}

1 A. Mleiki, S. Othmani, W. Cheikhrouhou-Koubaa, A. Cheikhrouhou and E. K. Hlil, Enhanced relative cooling power in Ga-doped $\mathrm{La}_{0.7}(\mathrm{Sr}, \mathrm{Ca})_{0.3} \mathrm{MnO}_{3}$ with ferromagneticlike canted state, $R S C A d v ., 2016,6,53602$.

2 Q. Zhang, L. Yin, W. Mi and X. Wang, Large Spatial Spin Polarization at Benzene/ $/ \mathrm{La}_{2 / 3} \mathrm{Sr}_{1 / 3} \mathrm{MnO}_{3}$ Spinterface: 
Toward Organic Spintronic Devices, J. Phys. Chem. C, 2016, 120, 6156.

3 R. Dinamarca, C. Sepúlveda, E. J. Delgado, O. Peña, J. L. G. Fierro and G. Pecchi, Electronic properties and catalytic performance for DME combustion of lanthanum manganites with partial B-site substitution, J. Catal., 2016, 338, 47.

4 S. Saha, K. Das, S. Bandyopadhyay and I. Das, A-site cationic disorder induced significantly large magnetoresistance in polycrystalline $\mathrm{La}_{0.2} \mathrm{Gd}_{0.5} \mathrm{Ba}_{0.3} \mathrm{MnO}_{3}$ compound, J. Magn. Magn. Mater., 2017, 442, 196.

5 Y. Tokura, Colossal Magnetoresistive Oxides, Gordon and Breach Science, New York, 2000.

6 T. Elovaara, H. Huhtinen, S. Majumdar and P. Paturi, Irreversible metamagnetic transition and magnetic memory in small-bandwidth manganite $\operatorname{Pr}_{1-x} \mathrm{Ca}_{x} \mathrm{MnO}_{3}(x=$ 0.0-0.5), J. Phys.: Condens. Matter, 2012, 24, 216002.

7 Y. Tokura, Critical features of colossal magnetoresistive manganites, Rep. Prog. Phys., 2006, 69, 797.

8 A.-M. Haghiri-Gosnet and J.-P. Renard, CMR manganites: physics, thin films and devices, J. Phys. D: Appl. Phys., 2003, 36, R127.

9 V. S. Kolat, T. Izgi, A. O. Kaya, N. Bayri, H. Gencer and S. Atalay, Metamagnetic transition and magnetocaloric effect in charge-ordered $\mathrm{Pr}_{0.68} \mathrm{Ca}_{0.32-x} \mathrm{Sr}_{x} \mathrm{MnO}_{3}(x=0,0.1$, $0.18,0.26$ and 0.32) compounds, J. Magn. Magn. Mater., 2010, 322, 427.

$10 \mathrm{~J}$. Dho and N. H. Hur, Thermal relaxation of field-induced irreversible ferromagnetic phase in Pr-doped manganites, Phys. Rev. B: Condens. Matter Mater. Phys., 2003, 67, 214414.

11 Y. Kashiwada, H. Fujishiro and M. Ibeke, Thermal conductivity of $\mathrm{Pr}_{0.65}\left(\mathrm{Ca}_{1-\mathrm{Z}} \mathrm{Sr}_{\mathrm{Z}}\right)_{0.35} \mathrm{MnO}_{3}$ under applied field, Physica B, 2003, 329, 924.

12 A. Biswas, T. Samanta, S. Banerjee and I. Das, Influence of charge ordering on magnetocaloric properties of nanocrystalline $\operatorname{Pr}_{0.65}\left(\mathrm{Ca}_{0.7} \mathrm{Sr}_{0.3}\right)_{0.35} \mathrm{MnO}_{3}$, Appl. Phys. Lett., 2008, 92, 012502.

13 S. Saha, K. Das, S. Bandyopadhyay and I. Das, A comparative study of magnetic field induced meta-magnetic transition in nanocrystalline and bulk $\operatorname{Pr}_{0.65}\left(\mathrm{Ca}_{0.7} \mathrm{Sr}_{0.3}\right)_{0.35} \mathrm{MnO}_{3}$ compound, J. Magn. Magn. Mater., 2017, 432, 271.

14 H. Yoshizawa, R. Kajimoto, H. Kawano, Y. Tomioka and Y. Tokura, Bandwidth-control-induced insulator-metal transition in $\operatorname{Pr}_{0.65}\left(\mathrm{Ca}_{1-\mathrm{y}} \mathrm{Sr}_{\mathrm{y}}\right)_{0.35} \mathrm{MnO}_{3}$ and $\mathrm{Pr}_{0.7} \mathrm{Ca}_{0.3} \mathrm{MnO}_{3}$, Phys. Rev. B: Condens. Matter Mater. Phys., 1997, 55, 2729.

$15 \mathrm{~J}$. Rodriguez-Carvajal, Recent advances in magnetic structure determination by neutron powder diffraction, Physica B, 1993, 192, 55.

16 S. Mollah, H. L. Huang, H. D. Yang, S. Pal, S. Taran and B. K. Chaudhuri, Non-adiabatic small-polaron hopping conduction in $\mathrm{Pr}_{0.65} \mathrm{Ca}_{0.35-x} \mathrm{Sr}_{x} \mathrm{MnO}_{3}$ perovskites above the metal-insulator transition temperature, J. Magn. Magn. Mater., 2004, 284, 383.

17 A. Mleiki, S. Othmani, W. Cheikhrouhou-Koubaa, M. Koubaa, A. Cheikhrouhou and E. K. Hlil, Effect of praseodymium doping on the structural, magnetic and magnetocaloric properties of $\mathrm{Sm}_{0.55-x} \mathrm{Pr}_{x} \mathrm{Sr}_{0.45} \mathrm{MnO}_{3}(0.1 \leq$ $x \leq 0.4)$ manganites, J. Alloys Compd., 2015, 645, 559.

18 H. Ben Khlifa, S. Othmani, I. Chaaba, S. Tarhouni, W. Cheikhrouhou-Koubaa, M. Koubaa, A. Cheikhrouhou and E. K. Hlil, Effect of K-doping on the structural, magnetic and magnetocaloric properties of $\mathrm{Pr}_{0.8} \mathrm{Na}_{0.2-x} \mathrm{~K}_{x} \mathrm{MnO}_{3}(0 \leq x \leq$ 0.15) manganites, J. Alloys Compd., 2016, 680, 388.

19 A. Mleiki, S. Othmani, W. Cheikhrouhou-Koubaa, M. Koubaa, A. Cheikhrouhou and E. K. Hlil, Effect of praseodymium doping on the structural, magnetic and magnetocaloric properties of $\mathrm{Sm}_{0.55} \mathrm{Sr}_{0.45} \mathrm{MnO}_{3}$ manganite, Solid State Commun., 2015, 223, 6.

20 I. Sfifir, A. Ezaami, W. Cheikhrouhou-Koubaa and A. Cheikhrouhou, Structural, magnetic and magnetocaloric properties in $\mathrm{La}_{0.7-x} \mathrm{Dy}_{x} \mathrm{Sr}_{0.3} \mathrm{MnO}_{3}$ manganites $(x=0.00$, 0.01 and 0.03), J. Alloys Compd., 2017, 696, 760.

21 A. Mleiki, S. Othmani, W. Cheikhrouhou-Koubaa, A. Cheikhrouhou and E. K. Hlil, Normal and inverse magnetocaloric effect and short-range ferromagnetic interaction in $(\mathrm{Pr}, \mathrm{Sm})_{0.5} \mathrm{Sr}_{0.5} \mathrm{MnO}_{3}$ phase separated manganite, J. Alloys Compd., 2016, 688, 1214.

22 R. P. Borges, F. Ott, R. M. Thomas, V. Skumryev, J. M. D. Coey, J. I. Arnaudas and L. Ranno, Field-induced transition in the paramagnetic state of $\mathrm{Sm}_{0.65} \mathrm{Sr}_{0.35} \mathrm{MnO}_{3}$ associated with magnetic clusters, Phys. Rev. B: Condens. Matter Mater. Phys., 1999, 60, 12847.

23 B. K. Banerjee, On a generalised approach to first and second order magnetic transitions, Phys. Lett., 1964, 12, 16.

24 A. Arrott and J. E. Noakes, Approximate equation of state for nickel near its critical temperature, Phys. Rev. Lett., 1967, 19, 786.

25 K. A. Gschneidner Jr, V. K. Pecharsky and A. O. Tsokol, Recent developments in magnetocaloric materials, Rep. Prog. Phys., 2005, 68, 1479.

26 A. Mleiki, R. M'nassri, W. Cheikhrouhou-Koubaa, A. Cheikhrouhou and E. K. Hlil, Structural characterization, magnetic, magnetocaloric properties and critical behavior in lacunar $\mathrm{La}_{0.5} \mathrm{Eu}_{0.2} \mathrm{Ba}_{0.2} \square_{0.1} \mathrm{MnO}_{3}$ nanoparticles, J. Alloys Compd., 2017, 727, 1203.

27 M. Wali, R. Skini, M. Khlifi, E. Dhahri and E. K. Hlil, A giant magnetocaloric effect with a tunable temperature transition close to room temperature in Na-deficient $\mathrm{La}_{0.8} \mathrm{Na}_{0.2-x} \mathrm{MnO}_{3}$ manganites, Dalton Trans., 2015, 44, 12796.

28 H. Omrani, M. Mansouri, W. C. Koubaa, M. Koubaa and A. Cheikhrouhou, Structural, magnetic and magnetocaloric investigations in $\operatorname{Pr}_{0.6-x} \mathrm{Er}_{x} \mathrm{Ca}_{0.1} \mathrm{Sr}_{0.3} \mathrm{MnO}_{3}(0 \leq x \leq 0.06)$ manganites, J. Alloys Compd., 2016, 688, 752.

29 S. Tarhouni, A. Mleiki, I. Chaaba, H. B. Khelifa, W. Cheikhrouhou-Koubaa, M. Koubaa and E. K. Hlil, Structural, magnetic and magnetocaloric properties of $\mathrm{Ag}$ doped $\mathrm{Pr}_{0.5} \mathrm{Sr}_{0.5-x} \mathrm{Ag}_{x} \mathrm{MnO}_{3}$ manganites $(0.0 \leq x \leq 0.4)$, Ceram. Int., 2017, 43, 133.

30 A. Ezaami, E. Sellami-Jmal, I. Chaaba, W. CheikhrouhouKoubaa, A. Cheikhrouhou and E. K. Hlil, Effect of elaborating method on magnetocaloric properties of $\mathrm{La}_{0.7} \mathrm{Ca}_{0.2} \mathrm{Ba}_{0.1} \mathrm{MnO}_{3}$ manganite, J. Alloys Compd., 2016, 685, 710 . 
31 A. Ezaami, W. Cheikhrouhou-Koubaa and A. Cheikhrouhou, Improvement of magnetocaloric properties over a large temperature range in $0.5 \mathrm{La}_{0.7} \mathrm{Ca}_{0.2} \mathrm{Sr}_{0.1} \mathrm{MnO}_{3} / 0.5 \mathrm{La}_{0 .}{ }_{7} \mathrm{Ca}_{0.15}$ $\mathrm{Sr}_{0.15} \mathrm{MnO}_{3}$ composite, J. Alloys Compd., 2017, 724, 851.

32 I. Messaoui, M. Kumaresavanji, K. Riahi, W. Cheikhrouhou Koubaa, M. Koubaa and A. Cheikhrouhou, Investigation on magnetic and magnetocaloric properties in the $\mathrm{Pb}$-doped manganites $\mathrm{La}_{0.78} \mathrm{Ca}_{0.22-x} \mathrm{~Pb}_{x} \mathrm{MnO}_{3}(x=0,0.05$ and 0.1 ), $J$. Alloys Compd., 2017, 693, 705.

33 V. Franco, A. Conde, J. M. Romero-Enrique and J. S. Blázquez, A universal curve for the magnetocaloric effect: an analysis based on scaling relations, J. Phys.: Condens. Matter, 2008, 20, 285207.

34 C. Romero-Muñiz, R. Tamura, S. Tanaka and V. Franco, Applicability of scaling behavior and power laws in the analysis of the magnetocaloric effect in second-order phase transition materials, Phys. Rev. B: Condens. Matter Mater. Phys., 2016, 94, 134401.

35 A. Biswas, S. Chandra, T. Samanta, B. Ghosh, S. Datta, M. H. Phan, A. K. Raychaudhuri, I. Das and H. Srikanth, Universality in the entropy change for the inverse magnetocaloric effect, Phys. Rev. B: Condens. Matter Mater. Phys., 2013, 87, 134420.

36 V. Franco, A. Conde, V. K. Pecharsky and K. A. Gschneidner, Field dependence of the magnetocaloric effect in Gd and $\left(\mathrm{Er}_{1-\mathrm{x}} \mathrm{Dy}_{\mathrm{x}}\right) \mathrm{Al}_{2}$ : Does a universal curve exist?, Europhys. Lett., 2007, 79, 47009.

37 C. M. Bonilla, J. Herrero-Albillos, F. Bartolome, L. M. Garcia, M. Parra-Borderıas and V. Franco, Universal behavior for magnetic entropy change in magnetocaloric materials: An analysis on the nature of phase transitions, Phys. Rev. B: Condens. Matter Mater. Phys., 2010, 81, 224424.

38 V. Franco and A. Conde, Scaling laws for the magnetocaloric effect in second order phase transitions: From physics to applications for the characterization of materials, Int. J. Refrig., 2010, 33, 465473.

39 V. Franco, R. Cabalero-Flores, A. Conde, Q. Y. Dong and H. W. Zhang, The influence of a minority magnetic phase on the field dependence of the magnetocaloric effect, $J$. Magn. Magn. Mater., 2009, 321, 1115.

40 H. Rahmouni, R. Jemai, M. Nouiri, N. Kallel, F. Rzigua, A. Selmi, K. Khirouni and S. Alaya, Admittance spectroscopy and complex impedance analysis of Timodified $\mathrm{La}_{0.7} \mathrm{Sr}_{0.3} \mathrm{MnO}_{3}$, J. Cryst. Growth, 2008, 310, 556.

41 S. R. Eilliot, Temperature dependence of ac. conductivity, Philos. Mag. B, 1978, 37, 553.

42 G. F. Pike, Phys. Rev. B: Solid State, 1972, 6, 1572.

43 N. F. Mott and E. A. Davis, Electronic Process in NonCrystalline Materials, Clarendon Press, Oxford, 1979.

44 E. V. Gopalan, K. A. Mallini, S. Saravanan, D. S. Kumar, Y. Yoshida and M. R. Anantharaman, Evidence for polaron conduction in nanostructured manganese ferrite, J. Phys. D: Appl. Phys., 2008, 41, 185005.

45 K. Funke, Jump relaxation in solid electrolytes, Prog. Solid State Chem., 1993, 22, 111.

46 J. Yang, X. Rong, D. Suter and Y. P. Sun, Electron paramagnetic resonance investigation of the electron- doped manganite $\mathrm{La}_{1-x} \mathrm{Te}_{x} \mathrm{MnO}_{3}(0.1 \leq x \leq 0.2)$, Phys. Chem. Chem. Phys., 2011, 13, 16343.

47 H. Rahmouni, B. Cherif, R. Jemai, A. Dhahri and K. Khirouni, Europium substitution for lanthanum in LaBaMnO-the structural and electrical properties of $\mathrm{La}_{0.7-x} \mathrm{Eu}_{x} \mathrm{Ba}_{0.3} \mathrm{MnO}_{3}$ perovskite, J. Alloys Compd., 2017, 690, 890.

48 H. Rahmouni, M. Smari, B. Cherif, E. Dhahri and K. Khirouni, Conduction mechanism, impedance spectroscopic investigation and dielectric behavior of $\mathrm{La}_{0.5} \mathrm{Ca}_{0.5-x} \mathrm{Ag}_{x} \mathrm{MnO}_{3}$ manganites with the composition below the concentration limit of silver solubility in perovskites $(0 \leq x \leq 0.2)$, Dalton Trans., 2015, 44, 10457.

49 A. Ben Jazia Kharrat, S. Moussa, N. Moutiaa, K. Khirouni and W. Boujelben, Structural, electrical and dielectric properties of Bi-doped $\operatorname{Pr}_{0.8-x} \mathrm{Bi}_{x} \mathrm{Sr}_{0.2} \mathrm{MnO}_{3}$ manganite oxides prepared by sol-gel process, J. Alloys Compd., 2017, 724, 389.

$50 \mathrm{~J}$. Suchanicz, The low-frequency dielectric relaxation $\mathrm{Na}_{0.5} \mathrm{Bi}_{0.5} \mathrm{TiO}_{3}$ ceramics, Mater. Sci. Eng., 1998, 55, 114.

$51 \mathrm{~J} . \mathrm{Hu}$ and $\mathrm{H}$. Qin, Giant magnetoimpedance effect in $\mathrm{La}_{0.7} \mathrm{Ca}_{0.3} \mathrm{MnO}_{3}$ under low magnetic fields, J. Magn. Magn. Mater., 2001, 231, 1.

52 S. Khadhraoui, A. Triki, S. Hcini, S. Zemni and M. Oumezzine, Variable-range-hopping conduction and dielectric relaxation in $\mathrm{Pr}_{0.6} \mathrm{Sr}_{0.4} \mathrm{Mn}_{0.6} \mathrm{Ti}_{0.4} \mathrm{O}_{3 \pm \delta}$ perovskite, J. Magn. Magn. Mater., 2014, 371, 69.

53 H. Rahmouni, A. Selmi, K. Khirouni and N. Kallel, Chromium effects on the transport properties in $\mathrm{La}_{0.7} \mathrm{Sr}_{0.3} \mathrm{Mn}_{1-x} \mathrm{Cr}_{x} \mathrm{O}_{3}$, J. Alloys Compd., 2012, 533, 93.

54 M. Smari, H. Rahmouni, N. Elghoul, I. Wallha, E. Dhahri and K. khirouni, Electric-dielectric properties and complex impedance analysis of $\mathrm{La}_{0.5} \mathrm{Ca}_{0.5-x} \mathrm{Ag}_{x} \mathrm{MnO}_{3}$ manganites, RSC Adv., 2014, 5, 2177.

55 P. S. Anantha and K. Haritharan, Structure and ionic transport studies of sodium borophosphate glassy system, Mater. Chem. Phys., 2005, 89, 428.

56 S. Kulkarni, B. M. Nagabhushana, N. Parvatikar, A. Koppalkar, C. Shivakumara and R. Damle, Dielectric and electrical studies of $\mathrm{Pr}^{3+}$ doped nano $\mathrm{CaSiO}_{3}$ perovskite ceramics, Mater. Res. Bull., 2014, 50, 197.

57 N. K. Singh, A. Panigrahi and R. N. P. Chaudhary, Structural and dielectric properties of $\mathrm{Ba}_{5} \mathrm{EuTi}_{3-x} \mathrm{Zr}_{x} \mathrm{Nb}_{7} \mathrm{O}_{30}$ relaxor ferroelectrics, Mater. Lett., 2001, 50, 1.

58 T. Kar and R. N. P. Chaudhary, Structural, dielectric and electrical conducting properties of $\mathrm{CsB}^{\prime} \mathrm{B}^{\prime \prime} \mathrm{O}_{6}\left(\mathrm{~B}^{\prime}=\mathrm{Nb}\right.$, Ta; $\left.\mathrm{B}^{\prime \prime}=\mathrm{W}, \mathrm{Mo}\right)$ ceramics, Mater. Sci. Eng., 2002, 90, 224.

59 R. W. Sillars, The properties of a dielectric containing semiconducting particles of various shapes, J. Inst. Electr. Eng., 1937, 80, 378.

60 N. V. Prasad, G. Prasad, T. Bhimasankaran, S. V. Suryanarayana and G. S. Kumar, Dielectric properties of cobalt doped cadmium oxalate crystals, Bull. Mater. Sci., 1996, 19, 639.

61 C. G. Koops, On the dispersion of resistivity and dielectric constant of some semiconductors at audio frequencies, Phys. Rev., 1951, 83, 121. 